LETTER TO THE EDITOR

\title{
Spectral gradient of the thermal millimetre continuum as a diagnostic for optical thickness in the solar atmosphere (Corrigendum)
}

\author{
A. S. Rodger and N. Labrosse \\ SUPA School of Physics and Astronomy, University of Glasgow, Glasgow G12 8QQ, UK \\ A\&A, 617, L6 (2018), https://doi .org/10.1051/0004-6361/201833848
}

Key words. radiation mechanisms: thermal - Sun: atmosphere - Sun: filaments, prominences - errata, addenda

In our article (Rodger \& Labrosse 2018) a small factor of $\frac{\pi}{\sqrt{3}}$ is missing from Eq. (1). The correct expression for the absorption coefficient from thermal bremsstrahlung should read:

$\kappa_{v}=1.77 \times 10^{-2} \frac{n_{\mathrm{e}}}{v^{2} T^{3 / 2}} g_{\mathrm{ff}} \sum_{i} Z_{i}^{2} n_{i}$.

The omission of the $\frac{\pi}{\sqrt{3}}$ term in the published study does not change our analysis or the conclusions which we draw.
The spectral gradient does not depend on it and thus the results from the models given in our analysis show simply a case with slightly lower optical thickness than would be expected.

\section{References}

Rodger, A. S., \& Labrosse, N. 2018, A\&A, 617, L6 